\title{
Microstructural Imaging of Shock-Recovered Berea Sandstone and Quartz Sand Using Scanning Electron Microscopy
}

\author{
M. Hilt, C.R. Hagelberg, R.P. Swift and W.J. Nellis
}

February 3, 2000

U.S. Department of Energy

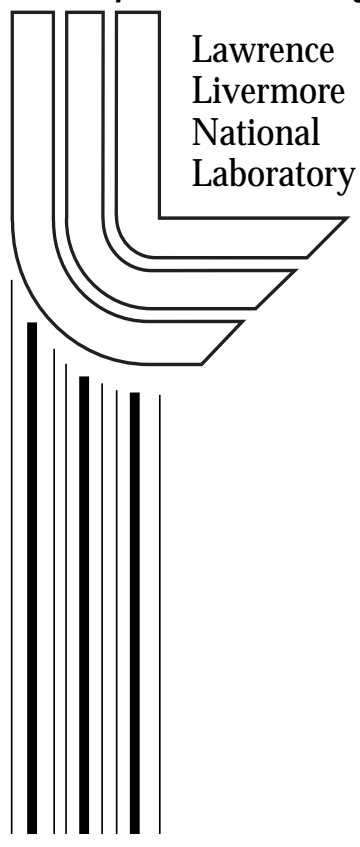




\section{DISCLAIMER}

This document was prepared as an account of work sponsored by an agency of the United States Government. Neither the United States Government nor the University of California nor any of their employees, makes any warranty, express or implied, or assumes any legal liability or responsibility for the accuracy, completeness, or usefulness of any information, apparatus, product, or process disclosed, or represents that its use would not infringe privately owned rights. Reference herein to any specific commercial product, process, or service by trade name, trademark, manufacturer, or otherwise, does not necessarily constitute or imply its endorsement, recommendation, or favoring by the United States Government or the University of California. The views and opinions of authors expressed herein do not necessarily state or reflect those of the United States Government or the University of California, and shall not be used for advertising or product endorsement purposes.

Work performed under the auspices of the U. S. Department of Energy by the University of California Lawrence Livermore National Laboratory under Contract W-7405-Eng-48.

This report has been reproduced directly from the best available copy.

Available to DOE and DOE contractors from the

Office of Scientific and Technical Information

P.O. Box 62, Oak Ridge, TN 37831

Prices available from (423) 576-8401

http://apollo.osti.gov/bridge/

Available to the public from the National Technical Information Service

U.S. Department of Commerce 5285 Port Royal Rd., Springfield, VA 22161 http://www.ntis.gov/

OR

Lawrence Livermore National Laboratory Technical Information Department's Digital Library http://www.llnl.gov/tid/Library.html 


\title{
Microstructural imaging of shock-recovered Berea sandstone and Quartz sand using Scanning Electron Microscopy
}

\author{
Michael HiltI ${ }^{1}$, Carl R. Hagelberg ${ }^{2}$, Robert P. Swift ${ }^{2}$, William J. Nellis ${ }^{1}$ \\ ${ }^{1}$ Lawrence Livermore National Laboratory, Livermore, California 94550 \\ ${ }^{2}$ Los Alamos National Laboratory, Los Alamos, New Mexico 87545
}

\begin{abstract}
A number of shock-recovery experiments have been performed on Berea sandstone for different conditions: dry, water-saturated, hydrostatically water-pressurized and Helium gas-pressurized. We also conducted experiments with purified quartz sand in dry and water-saturated conditions with a grain size between 212 to $250 \mu \mathrm{m}$ and 250 to $300 \mu \mathrm{m}$ to compare with damaged Berea sandstone. The shock stresses in the range between 1.2 to $9.8 \mathrm{GPa}$ were achieved by impacting projectiles accelerated by a single-stage light-gas gun. Different flyer plate thicknesses were used to produce different shock pulse durations. The water-pressurized sandstone targets were hydrostatically pressurized between 7.58-7.79 MPa, whereas the gas-pressure samples were pressurized to $27.5 \mathrm{MPa}$ using helium gas. The microstructural damage of all specimens is being investigated by using scanning electron microscopy (SEM) in order to determine differences for these conditions. In this report we will present the results of the systematic SEM investigations for each experiment. The scientific results and discussions including X-ray computed micro tomography and statistical analysis are presented elsewhere. Overall, we collected around 1600 SEM pictures, which are available in electronic form on Compact Disks (CDs). We also provide the results of the laser particle analysis on the CDs.
\end{abstract}

\section{Introduction}

Our motivation for this work comes in part from oil and gas recovery, where explosive shaped charges are used to perforate the wellbore casing to provide connectivity to the surrounding reservoir rock. The perforator jet causes deformation of each perforator tunnel. This so-called crushed zone (Krueger, 1986, Halleck, 1996) consist of fines from grain fragmentation, intergrain cracking and metal debris. The fines can significantly reduce the local permeability in the vicinity of the perforation and hinder recovery of hydrocarbon (Halleck, 1996 and 1997, McLeod, 1983). In this study, we examine grain fragmentation caused by short duration stress waves similar to perforation loading using a two-stage light-gas gun shown in Fig 1.

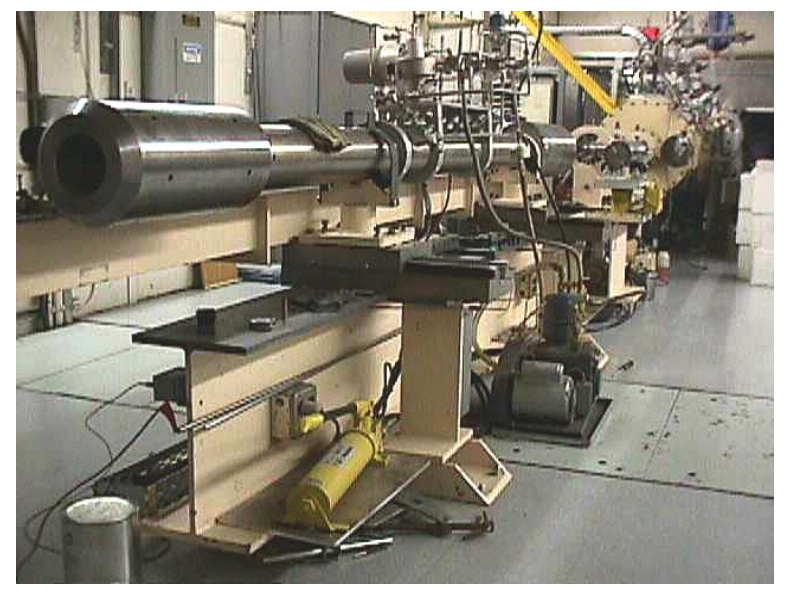

Fig. 1: Two-stage light-gas gun at Lawrence Livermore National Laboratory. 
Samples of Berea sandstone and pure quartz sand under dry, water-saturated, waterpressurized, and gas-pressurized conditions were subjected to well-defined impact loading using stress levels up to $10 \mathrm{GPa}$. Observations of grain damage were obtained from the recovered samples using the SEM. We are focused on basically two issues. First, to get a better understanding of shock induced grain damage and second, to provide quantitative data for correlation with grain/pore scale structure modeling of fragmentation caused by grain-to-grain interactions (Swift et al., 1998). A detailed description of computer modeling as well as scientific results and discussions of the shock-recovery experiments are reported elsewhere (Hagelberg et al., 2000ab, Hiltl et al., 2000abc, Swift et al., 2000ab).

\section{Material description}

For the shock recovery experiments we selected Berea sandstone, one of the most commonly used rock standards in petrophysical studies. This sandstone consists primarily of sub-angular, well-sorted quartz grains. Other mineral grains like feldspar, mica and clay aggregates (kaolinite which occurs primarily as flakes, montmorilonite and smectite) are present, together with accessory minerals namely zircon, rutile, magnetite and apatite. Calcite and dolomite cement the mineral grains. Pre-existing cracks were observed in some quartz grains of the undamaged material. We determined the bulk density of cylindrical samples by weighting and measuring several samples in air. The grain density was measured using a helium densitometer and the Archimedes method. The material properties of the sandstone used in this study are listed in Table. 1.

\begin{tabular}{|l|l|}
\hline Porosity: & $21.9 \%$ \\
\hline Bulk density: & $2.077 \mathrm{~g} / \mathrm{cm}^{3}$ \\
\hline Grain density: & $2.631 \mathrm{~g} / \mathrm{cm}^{3}$ \\
\hline Average grain size: & $0.15 \mathrm{~mm}$ \\
\hline
\end{tabular}

Tab. 1: Material properties of the investigated Berea sandstone.

The purified material for the quartz sand experiments was obtained from J. T. Barker Inc. We sieved the sand to a grain size of 212-250 $\mu \mathrm{m}$ and $250-300 \mu \mathrm{m}$, respectively. Material properties of quartz can be found, for example, in Lide, 1998.

\section{Experimental Setup}

The dry, water-saturated, water-pressurized and gas-pressurized recovery experiments have been conducted using a $4 \mathrm{~m}$ long single-stage light-gas gun with a bore diameter of $35 \mathrm{~mm}$. The experimental work was performed at Lawrence Livermore National Laboratory. All sandstone samples were confined in an aluminum 6061 (Lundergan and Herrmann, 1963, Mitchell and Nellis, 1981) capsule surrounded by a recovery fixture of the same material. The front face thickness of the capsule varies between 3 and $7 \mathrm{~mm}$. Figure 2 shows the target setup for the dry experiments. Aluminum 6061 was selected due to the similar shock impedance to Berea sandstone minimizing the impedance mismatch. Core plugs for the experiments were obtained from a Berea sandstone cylinder $(90 \times 70 \mathrm{~mm}$ ) by using a $22.4 \mathrm{~mm}$ and $25.4 \mathrm{~mm}$ diameter diamond coring drill. Water was used as a coolant to minimize the grain damage during the drilling process. Slices of approximately $5 \mathrm{~mm}$ and $15 \mathrm{~mm}$ were sawed from the cored material using a diamond saw. To achieve an accurate flatness the samples were ground and lapped. To avoid that grains pulling away from the edge during the cutting, grinding and lapping process, all samples were confined with a $1 \mathrm{~mm}$ thick aluminum 6061 ring. 


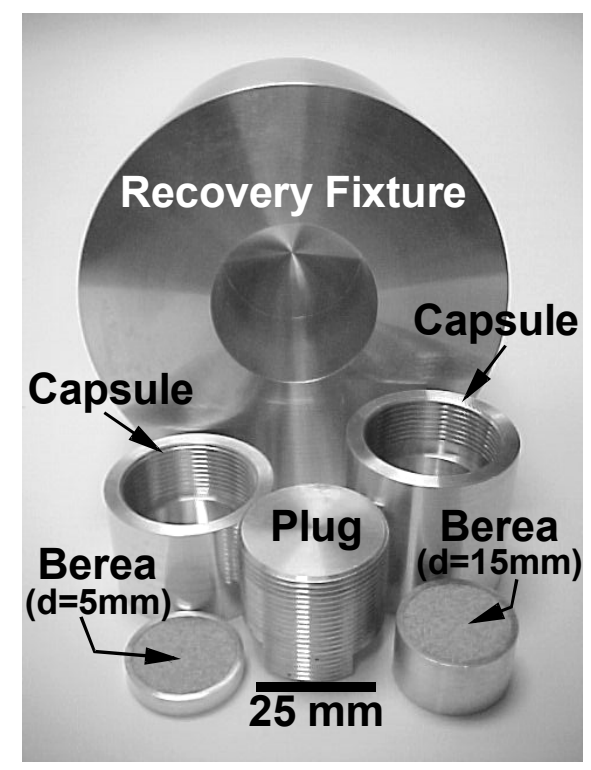

Fig. 2: Parts used to setup the target for dry conditions. Two different sample thicknesses $(5 \mathrm{~mm}$ and $15 \mathrm{~mm}$ ) were used.

The correct stress levels of shaped charges, typically between 1-10 GPa (Lichtenberger, 1995), were achieved by accelerating a projectile with expanding helium gas before impacting onto the capsule containing the sandstone target. The helium gas used to pressurize the gas breech is independent from the helium gas we used to gas-pressurize the Berea sandstone. The projectile consisted of a polycarbonate $\left(\operatorname{Lexan}^{\circledR}\right)$ carrier and a $32 \mathrm{~mm}$ in diameter flyer plate; either aluminum 1100, oxygen-free high-conductivity (OFHC) copper or PMMA (Polymethyl methacrylate). We chose two flyer plate thicknesses, approximately $3 \mathrm{~mm}$ and $6.25 \mathrm{~mm}$, to produce different shock pulse durations in the sandstone samples. Laser-diode measurments and X-ray photos of the projectile shortly before the impact were taken to determine the velocity of the flyer plate. The achieved impact pressures were calculated using the shock impedance matching method (Rice et al.(1958)) by knowing the impact velocity, the Hugoniot equations of state of the flyer plate material (Lundergan and Herrmann, 1963, Mitchell and Nellis, 1981, Marsh, 1979) and the container material. Computer simulations show an approximately $35 \%$ reduced stress levels in the sandstone material. Fig. 3 illustrates schematically the experimental configuration for the dry samples shortly before the impact. In this report we will list only the impact pressure produced by the accelerated flyer plate.

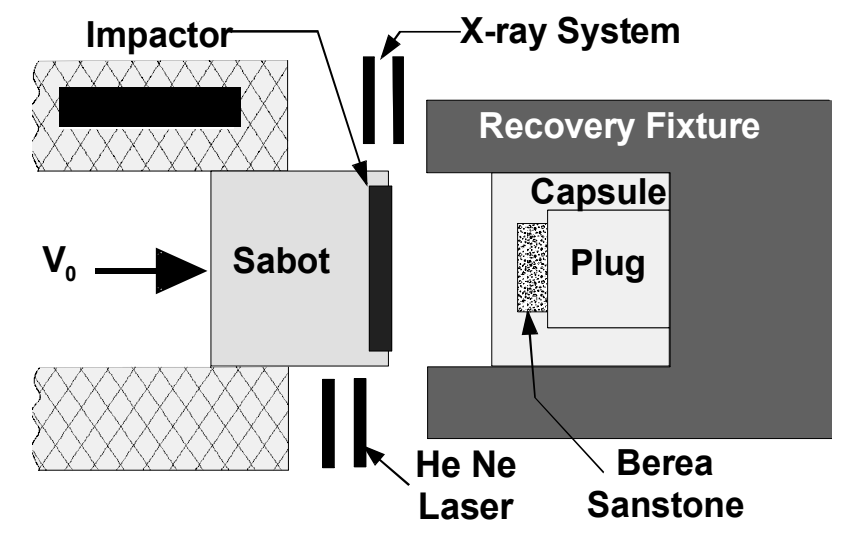

Fig. 3: Schematic setup for the experiments with dry Berea sandstone samples. 
To simulate downhole pressure conditions, we applied the water and gas pressure hydrostatically directly to the samples. This was realized by modifying the capsule used originally for the dry samples. Two stainless steel tubes with a diameter of $1.6 \mathrm{~mm}$ were fitted in the recovery fixture to the backside of the sealed capsule. A connection was made to two $1 \mathrm{~mm}$ boreholes, which end at the surface of the Berea target. A schematic drawing, that shows the experimental setup for water-saturated, water-pressurized and gas-pressurized conditions, is shown in Fig. 4.

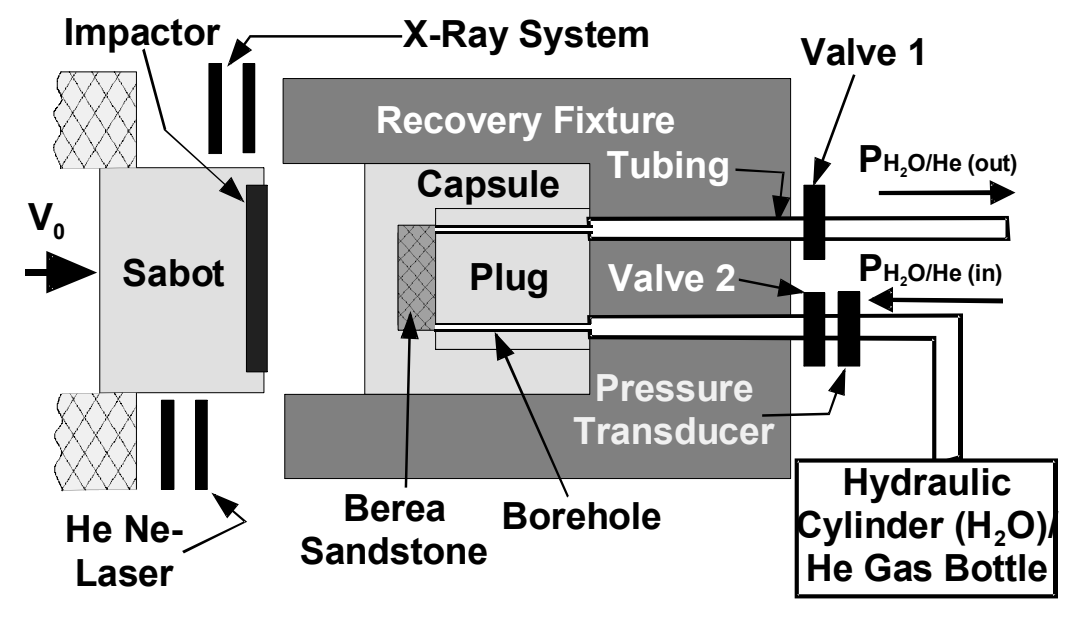

Fig. 4: Schematic setup for water-saturated, water-pressurized and gas-pressurized experiments.

Water-pressurizing the samples was done by pumping approximately $500 \mathrm{ml}$ of de-ionized water through the tubing and the sandstone to make sure that all air bubbles were out of the system. Valve 1 (Fig. 4) was closed and the sample was pressurized up to the desired pressures by using a hydraulic cylinder. The water pressure was measured and controlled by a calibrated pressure transducer. After reaching the correct hydrostatic water pressure valve 2 was closed to avoid any damage to the pressure transducer during the shock experiment. The projectile was then launched into the capsule using the gas gun. For the water-saturated experiments, water with a pressure of $690 \mathrm{kPa}$ was pushed through the targets to make sure that all air bubbles were out of the sandstone before closing valves 1 and 2 . We did the same procedure for the water-saturated experiments with quartz sand. For these experiments we used aluminum and stainless steel for the capsules as well as for the recovery fixtures. Figure 5 shows the experimental setup inside the gun tank.
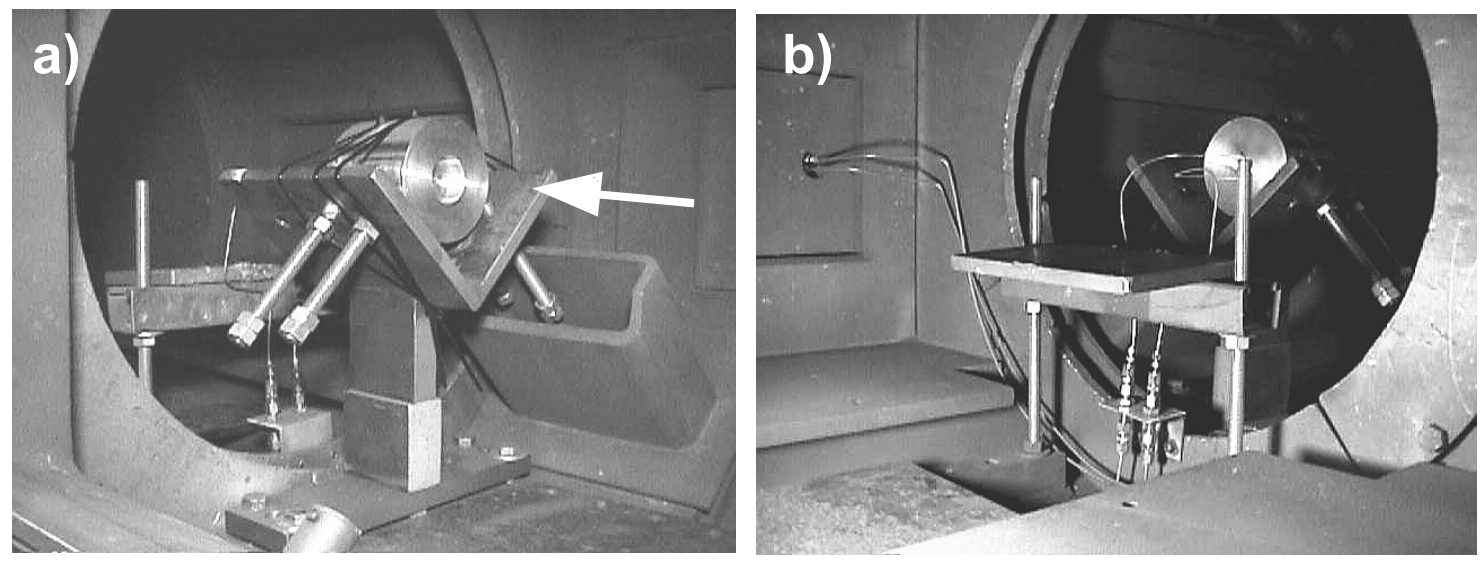

Fig 5: Target holder and Target inside the gun tank for water-saturated, water-pressurized and gas-pressurized conditions. a) front side (arrow indicates impact direction) and b) backside. 


\section{Sample preparation}

Preparing the recovered material plays a major role in the microstructural analysis. Therefore we tested several sample recovery methods and the following procedure is the most successful for our application. First, the capsules were removed from the recovery fixtures using a band saw with a fluid coolant. Then a diamond wire saw, having a wire diameter of $0.3 \mathrm{~mm}$, was used to cut each capsule into two halves. We preferred the wire saw because it makes a precise and smooth cut and therefore reduces the destruction of the original sandstone microstructure. After drying the two samples using an electrical oven at $60 \mathrm{C}$ for 1 hour, a very low viscose epoxy (EPO-TEK $301^{\mathrm{TM}}$ ) was pressed under vacuum into one of the two capsule halves. After the epoxy was hard and dry another cut was made, 1-2 mm away from the original cut, to get to the undisturbed material. The epoxied samples were then polished and a thin conductive layer of carbon was applied to the samples. This layer is required during SEM analysis to avoid build-up of electric charge due to the electron bombardment. The other half was used for laser particle size analysis.

Fig. 6 shows the sample preparation schematically.

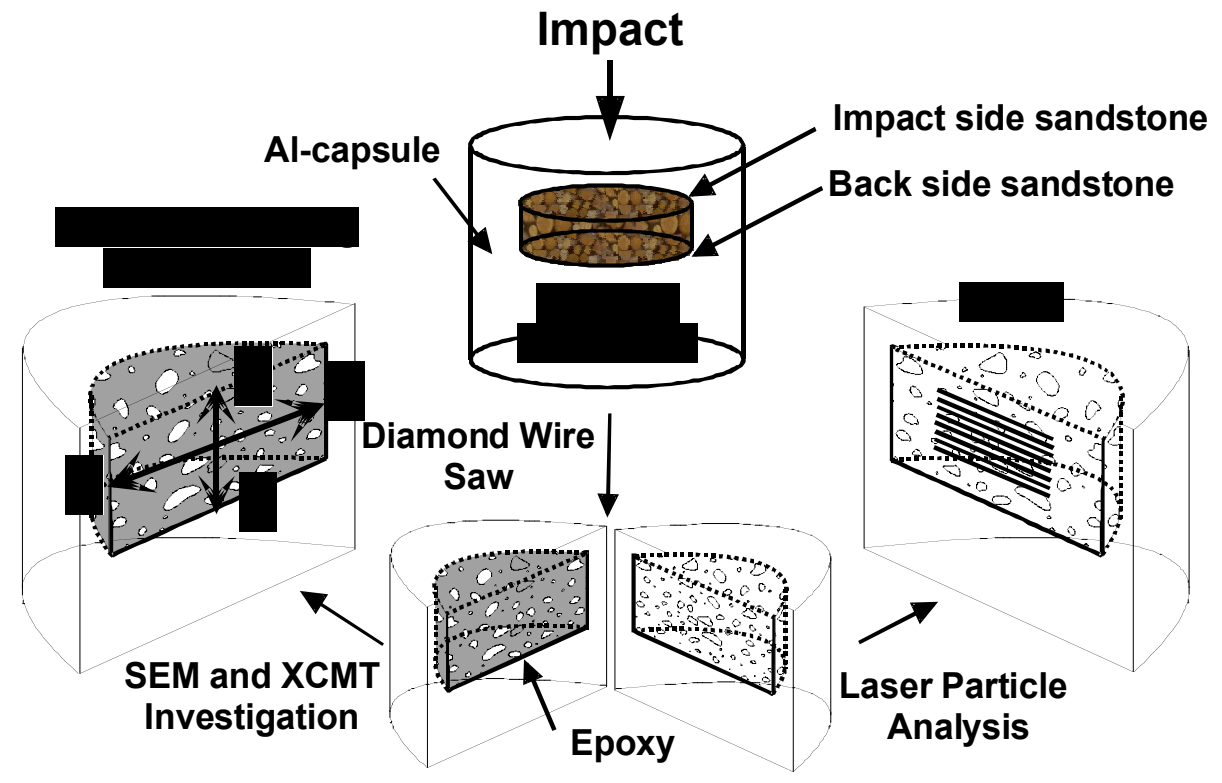

Fig. 6: Shocked capsule preparation technique for SEM and laser particle analysis.

\section{Laser particle size measurements}

Laser diffraction analyses using the unified scatter technique were performed at Pennsylvania State University. The machine employed for the analysis of the size distribution of the shockrecovered samples throughout this project is the Microtrac-X100 analyzer made by Honeywell Inc., FL/USA. All samples were measured in wet conditions using water as the fluid medium. Sandstone material was sliced from several depths (starting from the capsule impact side moving to capsule back side) with a scalpel (Fig. 6). A mortar and pestle was used to take the shocked Berea sandstone apart to get to the actual grain size. All aggregates with a size greater than 150 $\mu \mathrm{m}$ were sieved out. The sieved material was analyzed three times and the Microtrak software program was used to calculate an average grain size from the collected data. The calculations used to obtain results are based on the theory of optical diffraction by small suspended particles. The measurement system is based on Fourier optics. Laser light is shone through the sample cell, through which a constant flow of sandstone suspension is passed. The particles from the shocked Berea sandstone cause the light to be diffracted, depending on the size of the particle. Small particles diffract more than larger particles. The light is focused on to a detector. The flux pattern from the detector, averaged over a period of time, is deconvolved into a reading of particle 
sizes, calculated over discrete size ranges, the ranges given by the size of the detector areas. This measuring system assumes that the particles are completely opaque to the incoming radiation. A correction can be made to the flux pattern, allowing for refraction of light through the particles, provided the refractive index of the material is known. This system assumes that the particles are spherical. The calculation of the particle size is based on the fact that, for particles in the range of sizes corresponding to the wavelength of incident light, the difference in scattering of the vertically and horizontally polarized light is strongly dependent on the ratio of particle size to the wavelength of the incident light. The grain size distributions of the shocked Berea sandstone can be found on the attached CD.

\section{X-ray computed micro tomography}

Cores with a diameter of $2 \mathrm{~mm}$ and approximately $10 \mathrm{~mm}$ length were taken from the center part of the samples parallel to the axis of impact. The small Berea cores were analyzed using synchrotron computed micro tomography at Brookhaven National Laboratory with a voxel size of 3.6 and $6 \mu \mathrm{m}$, respectively. The XCMT process creates a stack of images each of which lies in the plane perpendicular to the cylinder axis. Figure 7 shows an undamaged Berea sandstone sample and provides an idea of the resolution obtained using XCMT. Figure 8a illustrates a 3DXMCT image of experiment 608 that was conducted under water-saturated conditions. The resolution for this particular sample was $3.6 \mu \mathrm{m}$. Fig. 8b shows the water-pressurized experiment 700 with a resolution of $6 \mu \mathrm{m}$. The scales for $\mathrm{x}, \mathrm{y}$ and $\mathrm{z}$ are given in pixels. With XCMT it is possible to distinguish the differences between undamaged Berea sandstone (Fig. 7) and the shock-recovered materials (Fig. 8). Porosity measurements of the two regions give values of 21.0 $\%$ and $13.3 \%$ for the undamaged and shocked regions, respectively. The use of X-ray computed microtomography is a useful complement and extension to other techniques like SEM and laser particle analysis. Because of the immense amount of XMCT data a detailed description of the analysis technique as well as a discussions will be reported separately (Hagelberg et al, 2000c).

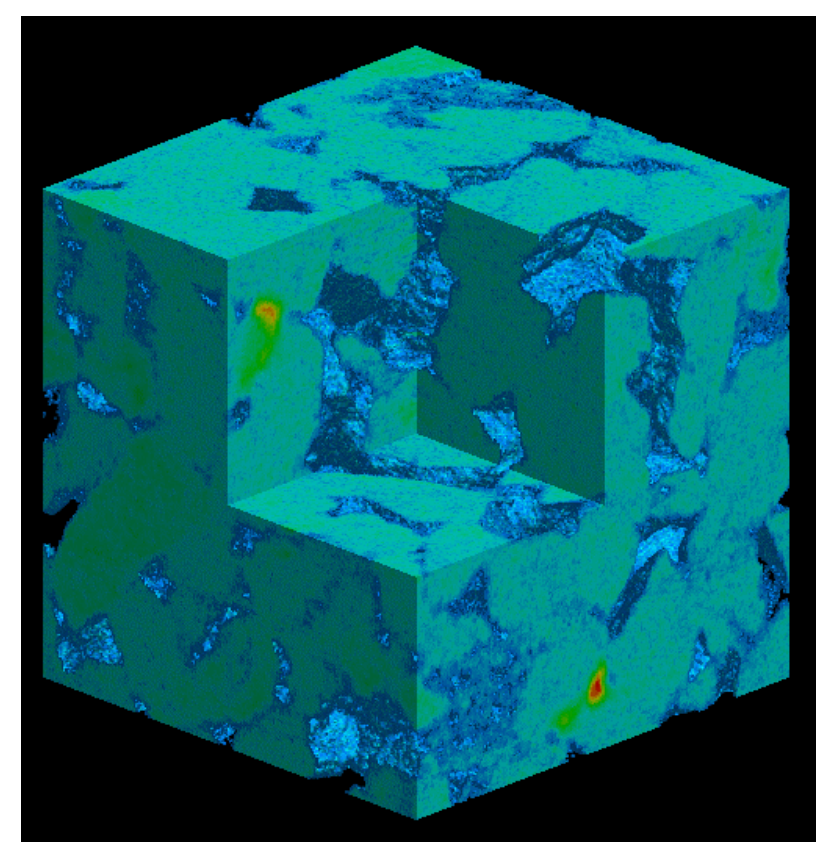

Fig. 7: 3-dimensional XCMT-image of undamaged Berea Sandstone. 

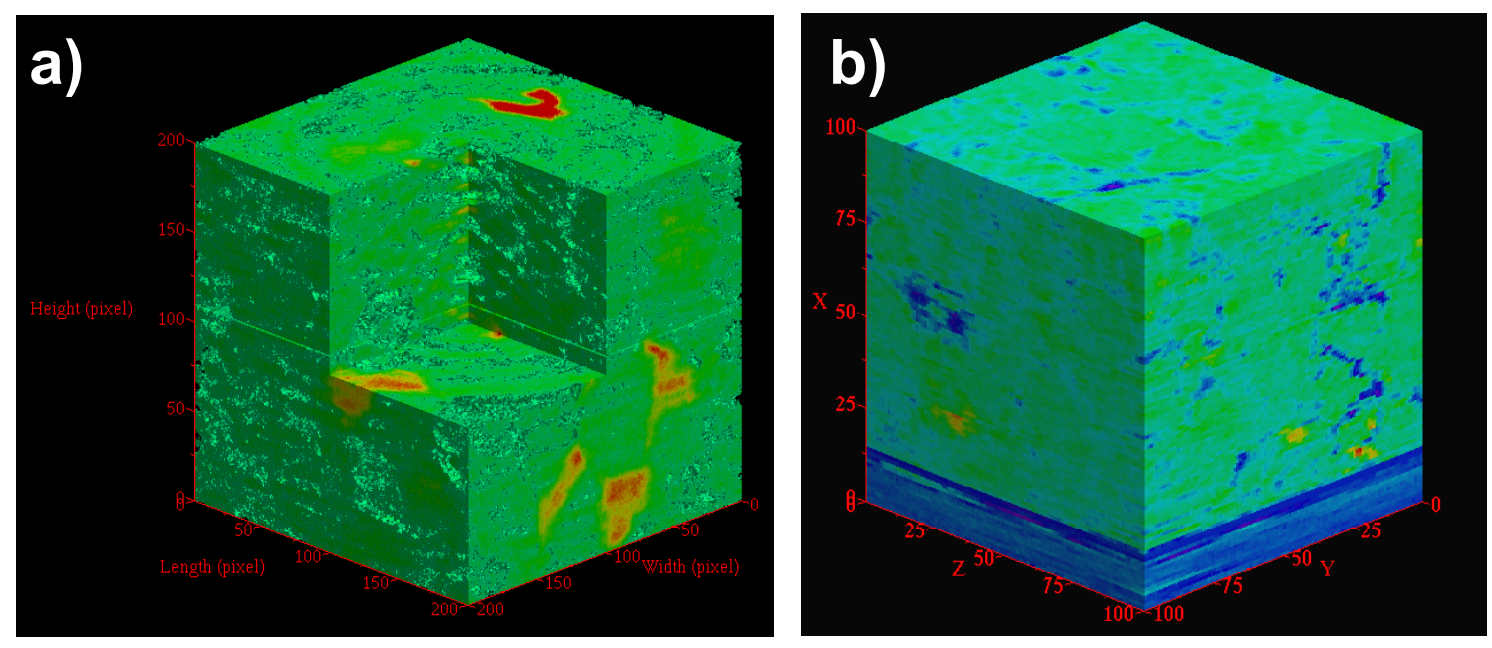

Fig. 8: 3-dimensional XCMT-image of a) experiment 608 (water-saturated) with a resolution of $3.6 \mu \mathrm{m}$ and b) experiment 700 (water-pressurized) with a resolution of $6 \mu \mathrm{m}$. (See Hagelberg et al, 2000c for more details).

\section{SEM information}

Systematic studies on the shock recovered samples were done at Los Alamos National Laboratory using a Noran Instruments automated digital electron microscope (ADEM) with a voltage of $15 \mathrm{kV}$ and a beam current of 100-300 picoamperes. We collected more then 1600 backscattered electron images (BSE) from all shock-recovered samples. The digital images were recorded mainly at a $1024 \times 1024$ pixel resolution, but some also at a $512 \times 512$ resolution using magnifications of $100 x$ and $400 x$. The gray levels of the images are related to the average atomic number of the minerals. The bigger the density of the material, the brighter it appears. However, different minerals (for example quartz, dolomite and sodium feldspar) can have similar average atomic numbers. To distinguish such minerals more accurately, X-ray analysis has to be used. The pores in the pictures appear dark, because they are filled with a low-density epoxy resin. We scanned each sample along the centerline from point $A$ to point $B$ (where $A$ is the impact side and $B$ the back side of the sandstone sample). On a few selected samples we did also a scan along the line $C-D$ to investigate edge effects. The way we scanned the samples is illustrated in Fig. 6 . The images are available in electronic form (tagged image format TIFF) on the attached CDs. The TIFF files are organized in directories having names corresponding to the experimental shot number. The two exceptions are UndamagedTiff and XrayMapsTiff that contain SEM images of the undamaged Berea and $x$-ray maps for elemental constituent analysis, respectively. Within the directories the TIFF files follow a general naming convention using the experiment number, magnification, and an identifying number. For example 744.401.01.tif refers to shot \# 744, magnification $401 \mathrm{x}$, image number 1 . For the undamaged Berea the naming convention uses „0k" to indicate the image comes from undamaged material. Unfortunately, the scanning sequence is not consistent throughout the data. For part of the data the scans start from the impact side of the sample and the rest start at the opposite end. It is easy to check by viewing the endpoint images. On occasion there are two sets of images of the same magnification, or two images saved of the same field of view. In these cases letters are appended to the filename constituents. For example, 608.100.01.tif and 608.100b.01.tif represent two images at 100x, but not of the same area, and saved at two different pixel resolutions (easily distinguished by file size). Or, for example, 696.100.01.tif and 696.100.01a.tif are two versions of the same image, with only a small change in SEM gain between the two. Such duplicate images are few and were kept for only completeness. There are six instances where the image was unsuccessfully saved from the SEM without the operators knowing (the images are blank or partially blank). These are 609.400.26.tif, 
700.400.mid1.tif, 700.400.mid4.tif, 701.400.mid2.tif, 743.10.04.tif, and 0k.400.13.tif. Any partial image was kept, but the blank images were discarded.

\section{Technical data of the shock-recovered samples}

In this section we will list important technical information for each of the shock-recovery experiments performed. Fig. 7 shows a schematic drawing of the capsule dimensions we will use.

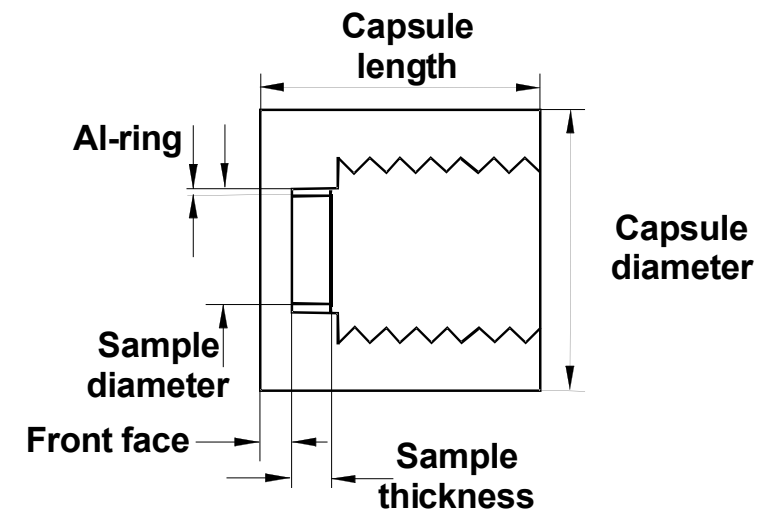

Fig. 7: Schematic drawing of the used capsules.

\section{Experiment 563:}

\begin{tabular}{|l|l|l|l|}
\hline Shot number: & 563 & Capsule dimensions: & $40 \mathrm{~mm}$ X 27.0 mm \\
\hline Sample diameter: & $22.4 \mathrm{~mm}$ & Capsule front face: & $3 \mathrm{~mm}$ \\
\hline Sample thickness: & $4.6 \mathrm{~mm}$ & Capsule material: & Aluminum 6061 \\
\hline Sample condition: & Dry & Ring material & Aluminum 6061 \\
\hline Flyer plate material: & OHFC copper & Gas breech pressure: & $22.10 \mathrm{MPa}$ \\
\hline Flyer plate diameter: & $32 \mathrm{~mm}$ & Barrel diameter: & $35 \mathrm{~mm}$ \\
\hline Flyer plate thickness: & $3.02 \mathrm{~mm}$ & Gap behind flyer: & no \\
\hline Flyer plate weight: & $21.98 \mathrm{~g}$ & X-ray taken: & yes \\
\hline Projectile material: & Lexan & Laser taken: & yes \\
\hline Projectile weight: & $51.1 \mathrm{~g}$ & Impact pressure: & $9.5 \mathrm{GPa}$ \\
\hline Projectile velocity & $820 \mathrm{~m} / \mathrm{s}$ & Date: & $05 / 27 / 1998$ \\
\hline Comments: & $\begin{array}{l}\text { SEM images: } 20 \text { total } \\
\text { 400X: } 20 \text { pictures named mh.b.90k.c. } 1 \text { - mh.b.90k.c.20 }\end{array}$ \\
\hline
\end{tabular}




\section{Experiment 564:}

\begin{tabular}{|l|l|l|l|}
\hline Shot number: & 564 & Capsule dimensions: & $40 \mathrm{~mm}$ X 27.0 mm \\
\hline Sample diameter: & $22.4 \mathrm{~mm}$ & Capsule front face: & $3 \mathrm{~mm}$ \\
\hline Sample thickness: & $4.95 \mathrm{~mm}$ & Capsule material: & Aluminum 6061 \\
\hline Sample condition: & Dry & Ring material & Aluminum 6061 \\
\hline Flyer plate material: & Aluminum 1100 & Gas breech pressure: & $17.9 \mathrm{MPa}$ \\
\hline Flyer plate diameter: & $32 \mathrm{~mm}$ & Barrel diameter: & $35 \mathrm{~mm}$ \\
\hline Flyer plate thickness: & $2.92 \mathrm{~mm}$ & Gap behind flyer: & no \\
\hline Flyer plate weight: & $6.43 \mathrm{~g}$ & X-ray taken: & yes \\
\hline Projectile material: & Lexan & Laser taken: & yes \\
\hline Projectile weight: & $35.6 \mathrm{~g}$ & Impact pressure: & $7.0 \mathrm{GPa}$ \\
\hline Projectile velocity & $870 \mathrm{~m} / \mathrm{s}$ & Date: & $5 / 27 / 1998$ \\
\hline Comments: & $\begin{array}{l}\text { SEM images: } 24 \text { total } \\
\text { 400X: } 24 \text { pictures named mh.70k.d.c1 - mh.70k.d.c24 }\end{array}$ \\
\hline
\end{tabular}

\section{Experiment 565:}

\begin{tabular}{|l|l|l|l|}
\hline Shot number: & 565 & Capsule dimensions: & $40 \mathrm{~mm}$ X 27.0 mm \\
\hline Sample diameter: & $22.4 \mathrm{~mm}$ & Capsule front face: & $3 \mathrm{~mm}$ \\
\hline Sample thickness: & $5.0 \mathrm{~mm}$ & Capsule material: & Aluminum 6061 \\
\hline Sample condition: & Dry & Ring material & Aluminum 6061 \\
\hline Flyer plate material: & Aluminum 1100 & Gas breech pressure: & $13.9 \mathrm{MPa}$ \\
\hline Flyer plate diameter: & $32 \mathrm{~mm}$ & Barrel diameter: & $35 \mathrm{~mm}$ \\
\hline Flyer plate thickness: & $2.97 \mathrm{~mm}$ & Gap behind flyer: & no \\
\hline Flyer plate weight: & $6.55 \mathrm{~g}$ & X-ray taken: & yes \\
\hline Projectile material: & Lexan & Laser taken: & yes \\
\hline Projectile weight: & $35.7 \mathrm{~g}$ & Impact pressure: & $6.1 \mathrm{GPa}$ \\
\hline Projectile velocity & $760 \mathrm{~m} / \mathrm{s}$ & Date: & $05 / 28 / 1998$ \\
\hline Comments: & $\begin{array}{l}\text { SEM images: } 26 \text { total } \\
\text { 400X: } 26 \text { pictures named mh.b.50k.c1 - mh.b.50k.c26. }\end{array}$ \\
\hline
\end{tabular}

\section{Experiment 577:}

\begin{tabular}{|l|l|l|l|}
\hline Shot number: & 577 & Capsule dimensions: & $40 \mathrm{~mm}$ X 27.0 mm \\
\hline Sample diameter: & $22.4 \mathrm{~mm}$ & Capsule front face: & $3 \mathrm{~mm}$ \\
\hline Sample thickness: & $4.64 \mathrm{~mm}$ & Capsule material: & Aluminum 6061 \\
\hline Sample condition: & Dry & Ring material & Aluminum 6061 \\
\hline Flyer plate material: & Aluminum 1100 & Gas breech pressure: & $11.1 \mathrm{MPa}$ \\
\hline Flyer plate diameter: & $32 \mathrm{~mm}$ & Barrel diameter: & $35 \mathrm{~mm}$ \\
\hline Flyer plate thickness: & $3.02 \mathrm{~mm}$ & Gap behind flyer: & no \\
\hline Flyer plate weight: & $6.36 \mathrm{~g}$ & X-ray taken: & yes \\
\hline Projectile material: & Lexan & Laser taken: & yes \\
\hline Projectile weight: & $32.0 \mathrm{~g}$ & Impact pressure: & $6.0 \mathrm{GPa}$ \\
\hline Projectile velocity & $750 \mathrm{~m} / \mathrm{s}$ & Date: & $08 / 07 / 1998$ \\
\hline Comments: & $\begin{array}{l}\text { The sample was water-pressurized to 7.24 GPa using the hydraulic } \\
\text { cylinder. Note that these samples was gold coated for the SEM. } \\
\text { SEM images: } 21 \text { total } \\
\text { 400X: 21 picture named mh.b.50k.w.g1 - mh.b.50k.w.g26 }\end{array}$ \\
\hline
\end{tabular}




\section{Experiment 579:}

\begin{tabular}{|l|l|l|l|}
\hline Shot number: & 579 & Capsule dimensions: & $40 \mathrm{~mm}$ X 27.0 mm \\
\hline Sample diameter: & $22.4 \mathrm{~mm}$ & Capsule front face: & $3 \mathrm{~mm}$ \\
\hline Sample thickness: & $4.61 \mathrm{~mm}$ & Capsule material: & Aluminum 6061 \\
\hline Sample condition: & Dry & Ring material & Aluminum 6061 \\
\hline Flyer plate material: & Aluminum 1100 & Gas breech pressure: & $17.2 \mathrm{MPa}$ \\
\hline Flyer plate diameter: & $32 \mathrm{~mm}$ & Barrel diameter: & $35 \mathrm{~mm}$ \\
\hline Flyer plate thickness: & $3.02 \mathrm{~mm}$ & Gap behind flyer: & no \\
\hline Flyer plate weight: & $6.43 \mathrm{~g}$ & X-ray taken: & yes \\
\hline Projectile material: & Lexan & Laser taken: & yes \\
\hline Projectile weight: & $35.5 \mathrm{~g}$ & Impact pressure: & $6.9 \mathrm{GPa}$ \\
\hline Projectile velocity & $850 \mathrm{~m} / \mathrm{s}$ & Date: & $08 / 17 / 1998$ \\
\hline Comments: & $\begin{array}{l}\text { The sample was water-pressurized to 7.54 GPa using the hydraulic } \\
\text { cylinder. Note that these sample was gold coated for the SEM. } \\
\text { SEM images: } 18 \text { total } \\
\text { 400X: } 18 \text { pictures named mh.b.70k.w.g1 - mh.b.70k.w.g18 }\end{array}$ \\
\hline
\end{tabular}

\section{Experiment 605:}

\begin{tabular}{|l|l|l|l|}
\hline Shot number: & 605 & Capsule dimensions: & $40 \mathrm{~mm} \times 27.0 \mathrm{~mm}$ \\
\hline Sample diameter: & $22.4 \mathrm{~mm}$ & Capsule front face: & $5 \mathrm{~mm}$ \\
\hline Sample thickness: & $5.0 \mathrm{~mm}$ & Capsule material: & Aluminum 6061 \\
\hline Sample condition: & Dry & Ring material & Aluminum 6061 \\
\hline Flyer plate material: & Aluminum 1100 & Gas breech pressure: & $13.79 \mathrm{MPa}$ \\
\hline Flyer plate diameter: & $32 \mathrm{~mm}$ & Barrel diameter: & $35 \mathrm{~mm}$ \\
\hline Flyer plate thickness: & $3.03 \mathrm{~mm}$ & Gap behind flyer: & no \\
\hline Flyer plate weight: & $6.67 \mathrm{~g}$ & X-ray taken: & yes \\
\hline Projectile material: & Lexan & Laser taken: & yes \\
\hline Projectile weight: & $35.3 \mathrm{~g}$ & Impact pressure: & $6.3 \mathrm{GPa}$ \\
\hline Projectile velocity & $780 \mathrm{~m} / \mathrm{s}$ & Date: & $11 / 02 / 1998$ \\
\hline Comments: & SEM pictures: 8 total \\
& $\begin{array}{l}100 X: 3 \text { pictures named } 605.100 .01-04 \\
\end{array}$ & 400X: 4 pictures impact side named 605.400.top1-4 \\
\hline
\end{tabular}




\section{Experiment 606:}

\begin{tabular}{|c|c|c|c|}
\hline Shot number: & 606 & Capsule dimensions: & $40 \mathrm{~mm} \times 33.8 \mathrm{~mm}$ \\
\hline Sample diameter: & $22.4 \mathrm{~mm}$ & Capsule front face: & $3 \mathrm{~mm}$ \\
\hline Sample thickness: & $14.8 \mathrm{~mm}$ & Capsule material: & Aluminum 6061 \\
\hline Sample condition: & Dry & Ring material & Aluminum 6061 \\
\hline Flyer plate material: & Aluminum 1100 & Gas breech pressure: & $13.79 \mathrm{MPa}$ \\
\hline Flyer plate diameter: & $32 \mathrm{~mm}$ & Barrel diameter: & $35 \mathrm{~mm}$ \\
\hline Flyer plate thickness: & $6.25 \mathrm{~mm}$ & Gap behind flyer: & yes \\
\hline Flyer plate weight: & $13.64 \mathrm{~g}$ & X-ray taken: & yes \\
\hline Projectile material: & Lexan & Laser taken: & yes \\
\hline Projectile weight: & $40.6 \mathrm{~g}$ & Impact pressure: & $6.1 \mathrm{GPa}$ \\
\hline Projectile velocity & $760 \mathrm{~m} / \mathrm{s}$ & Date: & $11 / 02 / 1998$ \\
\hline Comments: & \multicolumn{3}{|c|}{$\begin{array}{l}\text { SEM images: } 106 \text { total } \\
\text { 100X: } 16 \text { pictures named } 606.100 .01-606.100 .16 \\
\text { (side by side vertical scans, bottom to top, then top to bot.) } \\
\text { 100X: } 46 \text { pictures named } 606.100 \text { b. } 01-606.100 \text { b.46 } \\
\text { (vertical scan bottom to top, then three horizontal left to right } \\
\text { top, middle, and bottom) } \\
\text { 100X: } 2 \text { pictures named } 606.100 \text { p.01, } 606.100 p .02 \\
\text { ( top and bottom at } 512 \text { resolution) } \\
\text { 400X: } 12 \text { pictures named } 606.400 . r 1.01-606.400 . r 3.04 \\
\text { (mosaics of four at top, middle and bottom) } \\
\text { 400X: } 28 \text { pictures named } 606.401 .01-606.401 .28 \\
\text { (vertical scan bottom to top) } \\
\text { 400X: } 606.401 . \text { scale (intended to record a zoom, but didn't work) } \\
\text { Note: } 606 \text { 100x transect } 1:(01-08) \text { bottom to top; } \\
\text { transect } 2:(09-16) \text { top to bottom. }\end{array}$} \\
\hline
\end{tabular}

\section{Experiment 607:}

\begin{tabular}{|l|l|l|l|}
\hline Shot number: & 607 & Capsule dimensions: & $40 \mathrm{~mm}$ X 27.0 mm \\
\hline Sample diameter: & $22.4 \mathrm{~mm}$ & Capsule front face: & $3 \mathrm{~mm}$ \\
\hline Sample thickness: & $14.9 \mathrm{~mm}$ & Capsule material: & Aluminum 6061 \\
\hline Sample condition: & Dry & Ring material & Aluminum 6061 \\
\hline Flyer plate material: & Aluminum 1100 & Gas breech pressure: & $3.49 \mathrm{MPa}$ \\
\hline Flyer plate diameter: & $32 \mathrm{~mm}$ & Barrel diameter: & $35 \mathrm{~mm}$ \\
\hline Flyer plate thickness: & $3.02 \mathrm{~mm}$ & Gap behind flyer: & yes \\
\hline Flyer plate weight: & $6.63 \mathrm{~g}$ & X-ray taken: & yes \\
\hline Projectile material: & Lexan & Laser taken: & yes \\
\hline Projectile weight: & $33.7 \mathrm{~g}$ & Impact pressure: & $3.1 \mathrm{GPa}$ \\
\hline Projectile velocity & $390 \mathrm{~m} / \mathrm{s}$ & Date: & $11 / 03 / 1998$ \\
\hline Comments: & SEM images: 48 total \\
& $100 X: 8$ pictures named $607.100 .01-607.100 .08$ (bottom to top) \\
& $100 X: 8$ pictures named 607.100b.01-607.100b.08 \\
& (bottom to top, slightly different track) \\
& 400X: 32 pictures named 607.401.01 - 607.401.32 (bot. to top) \\
\hline
\end{tabular}




\section{Experiment 608:}

\begin{tabular}{|c|c|c|c|}
\hline Shot number: & 608 & Capsule dimensions: & $40 \mathrm{~mm} \times 27.0 \mathrm{~mm}$ \\
\hline Sample diameter: & $22.4 \mathrm{~mm}$ & Capsule front face: & $3 \mathrm{~mm}$ \\
\hline Sample thickness: & $14.6 \mathrm{~mm}$ & Capsule material: & Aluminum 6061 \\
\hline Sample condition: & Dry & Ring material & Aluminum 6061 \\
\hline Flyer plate material: & Aluminum 1100 & Gas breech pressure: & $13.1 \mathrm{MPa}$ \\
\hline Flyer plate diameter: & $32 \mathrm{~mm}$ & Barrel diameter: & $35 \mathrm{~mm}$ \\
\hline Flyer plate thickness: & $3.02 \mathrm{~mm}$ & Gap behind flyer: & yes \\
\hline Flyer plate weight: & $6.56 \mathrm{~g}$ & X-ray taken: & yes \\
\hline Projectile material: & Lexan & Laser taken: & yes \\
\hline Projectile weight: & $33.8 \mathrm{~g}$ & Impact pressure: & $6.4 \mathrm{GPa}$ \\
\hline Projectile velocity & $800 \mathrm{~m} / \mathrm{s}$ & Date: & $11 / 03 / 1998$ \\
\hline Comments: & \multicolumn{3}{|c|}{$\begin{array}{l}\text { SEM images: } 125 \text { total } \\
\text { 100X: } 7 \text { pictures named } 608.100 .01-608.100 .07 \\
\text { (vertical scan top to bottom) } \\
\text { 100X: } 1 \text { picture named } 608.100 a .01 \text { (same as } 608.100 .01 \text { with } \\
\text { (a different gain setting) } \\
\text { 100X: } 608.100 \mathrm{~b} .01608 .100 \mathrm{~b} .43 \text { (bottom to top plus three } \\
\text { horizontal scans, left to right at top, middle, and bottom) } \\
\text { 100X: } 608.100 \mathrm{H} 1.01 \text { ( } 1024 \text { resolution, bottom), } 608.100 \mathrm{~L} 1.01- \\
608.100 \mathrm{~L} 1.19 \text { ( } 512 \text { resolution vertical scan bottom to top } \\
\text { plus horizontal scan along bottom) } \\
\text { 400X: } 608.400 . \mathrm{r} 1.01 \text { through } 608.400 . \mathrm{r} 6.04 \text { ( } 24 \text { images) } \\
\text { (r1 and r4: top; } \mathrm{r} 2 \text { and } \mathrm{r} 5 \text { : middle; } \mathrm{r} 3 \text { and } \mathrm{r} 6 \text { bottom) }\end{array}$} \\
\hline
\end{tabular}

\section{Experiment 609:}

\begin{tabular}{|c|c|c|c|}
\hline Shot number: & 609 & Capsule dimensions: & $40 \mathrm{~mm} \times 27.0 \mathrm{~mm}$ \\
\hline Sample diameter: & $22.4 \mathrm{~mm}$ & Capsule front face: & $3 \mathrm{~mm}$ \\
\hline Sample thickness: & $14.7 \mathrm{~mm}$ & Capsule material: & Aluminum 6061 \\
\hline Sample condition: & Dry & Ring material & Aluminum 6061 \\
\hline Flyer plate material: & Aluminum 1100 & Gas breech pressure: & $22.06 \mathrm{MPa}$ \\
\hline Flyer plate diameter: & $32 \mathrm{~mm}$ & Barrel diameter: & $35 \mathrm{~mm}$ \\
\hline Flyer plate thickness: & $2.98 \mathrm{~mm}$ & Gap behind flyer: & yes \\
\hline Flyer plate weight: & $21.47 \mathrm{~g}$ & X-ray taken: & yes \\
\hline Projectile material: & Lexan & Laser taken: & yes \\
\hline Projectile weight: & $48.50 \mathrm{~g}$ & Impact pressure: & $9.8 \mathrm{GPa}$ \\
\hline Projectile velocity & $850 \mathrm{~m} / \mathrm{s}$ & Date: & $11 / 03 / 1998$ \\
\hline Comments: & \multicolumn{3}{|c|}{$\begin{array}{l}\text { SEM images: } 34 \text { total } \\
\text { 100X: } 7 \text { pictures named } 609.100 .01-609.100 .07 \text { (bottom to top) } \\
\text { 400X: } 28 \text { pictures named } 609.400 .01-609.400 .28 \\
\quad \text { (bottom to top) }\end{array}$} \\
\hline
\end{tabular}




\section{Experiment 610:}

\begin{tabular}{|l|l|l|l|}
\hline Shot number: & 610 & Capsule dimensions: & $40 \mathrm{~mm}$ X 27.0 mm \\
\hline Sample diameter: & $22.4 \mathrm{~mm}$ & Capsule front face: & $5 \mathrm{~mm}$ \\
\hline Sample thickness: & $5.0 \mathrm{~mm}$ & Capsule material: & Aluminum 6061 \\
\hline Sample condition: & Water-pressurized & Ring material & Aluminum 6061 \\
\hline Flyer plate material: & OHFC copper & Gas breech pressure: & $22.06 \mathrm{MPa}$ \\
\hline Flyer plate diameter: & $32 \mathrm{~mm}$ & Barrel diameter: & $35 \mathrm{~mm}$ \\
\hline Flyer plate thickness: & $2.98 \mathrm{~mm}$ & Gap behind flyer: & no \\
\hline Flyer plate weight: & $21.67 \mathrm{~g}$ & X-ray taken: & yes \\
\hline Projectile material: & Lexan & Laser taken: & yes \\
\hline Projectile weight: & $50.3 \mathrm{~g}$ & Impact pressure: & $9.7 \mathrm{GPa}$ \\
\hline Projectile velocity & $830 \mathrm{~m} / \mathrm{s}$ & Date: & $11 / 04 / 1998$ \\
\hline Comments: & $\begin{array}{l}\text { The sample was water-pressurized to 7.31 GPa using the hydraulic } \\
\text { cylinder. } \\
\text { SEM pictures: 7 total } \\
\text { 100X: 3 pictures named 610.100.01-03 (top, middle, bottom) } \\
\text { 400X: 4 pictures impact side named 610.400.top1-4 }\end{array}$ \\
\hline
\end{tabular}

\section{Experiment 611:}

\begin{tabular}{|c|c|c|c|}
\hline Shot number: & 611 & Capsule diameter: & $40 \mathrm{~mm} \times 33.8 \mathrm{~mm}$ \\
\hline Sample diameter: & $22.4 \mathrm{~mm}$ & Capsule front face: & $3 \mathrm{~mm}$ \\
\hline Sample thickness: & $14.5 \mathrm{~mm}$ & Capsule material: & Aluminum 6061 \\
\hline Sample condition: & Water-saturated & Ring material & Aluminum 6061 \\
\hline Flyer plate material: & Aluminum 1100 & Gas breech pressure: & $13.79 \mathrm{MPa}$ \\
\hline Flyer plate diameter: & $32 \mathrm{~mm}$ & Barrel diameter: & $35 \mathrm{~mm}$ \\
\hline Flyer plate thickness: & $6.21 \mathrm{~mm}$ & Gap behind flyer: & yes \\
\hline Flyer plate weight: & $13.48 \mathrm{~g}$ & X-ray taken: & yes \\
\hline Projectile material: & Lexan & Laser taken: & yes \\
\hline Projectile weight: & $40.5 \mathrm{~g}$ & Impact pressure: & $6.1 \mathrm{GPa}$ \\
\hline Projectile velocity & $760 \mathrm{~m} / \mathrm{s}$ & Date: & $11 / 04 / 1998$ \\
\hline Comments: & \multicolumn{3}{|c|}{$\begin{array}{l}\text { SEM images: } 94 \text { total } \\
\text { 100X: } 46 \text { pictures named } 611.100 .01-46 \text { (vertical scan bottom } \\
\text { to top, plus } 3 \text { horizontal scans left to right top, middle, } \\
\text { and bottom) } \\
\text { 100X: } 7 \text { pictures named } 611.100 a .01-07 \text { ( } 512 \text { resolution vertical } \\
\text { scan bottom to top) } \\
\text { 100X: } 2 \text { pictures named } 611.100 \mathrm{~b} .01 \text { (top and } 611.100 \mathrm{~b} .02 \text { (bot) } \\
400 \mathrm{X}: 27 \text { pictures named } 611.400 .01-26 \text { (vertical scan bottom to } \\
\text { top) } \\
400 \mathrm{X} \text { : } 12 \text { pictures named } 611.402 . \mathrm{r} 1 \mathrm{q} 1-611.402 . \mathrm{r} 3 \mathrm{q} 4 \text { ( } 3 \text { regions - } \\
\text { top, middle, and bottom- } 4 \text { quadrants each to make } \\
\text { mosaics) }\end{array}$} \\
\hline
\end{tabular}




\section{Experiment 613:}

\begin{tabular}{|l|l|l|l|}
\hline Shot number: & 613 & Capsule dimensions: & $40 \mathrm{~mm}$ X 27.0 mm \\
\hline Sample diameter: & $22.4 \mathrm{~mm}$ & Capsule front face: & $3 \mathrm{~mm}$ \\
\hline Sample thickness: & $14.7 \mathrm{~mm}$ & Capsule material: & Aluminum 6061 \\
\hline Sample condition: & Water-saturated & Ring material & Aluminum 6061 \\
\hline Flyer plate material: & Aluminum 1100 & Gas breech pressure: & $3.45 \mathrm{MPa}$ \\
\hline Flyer plate diameter: & $32 \mathrm{~mm}$ & Barrel diameter: & $35 \mathrm{~mm}$ \\
\hline Flyer plate thickness: & $3.02 \mathrm{~mm}$ & Gap behind flyer: & yes \\
\hline Flyer plate weight: & $6.58 \mathrm{~g}$ & X-ray taken: & yes \\
\hline Projectile material: & Lexan & Laser taken: & yes \\
\hline Projectile weight: & $33.8 \mathrm{~g}$ & Impact pressure: & $3.1 \mathrm{GPa}$ \\
\hline Projectile velocity & $390 \mathrm{~m} / \mathrm{s}$ & Date: & $11 / 05 / 1998$ \\
\hline Comments: & $\begin{array}{l}\text { SEM images: } 46 \text { total } \\
100 X: 2 \text { pictures named 613.100a.01-02 (top and bottom) }\end{array}$ \\
& $\begin{array}{l}100 \mathrm{X}: 10 \text { pictures named 613.100b.01 - 613.100b.10 (vertical } \\
\text { scan bottom to top, plus upper right and lower right corners) }\end{array}$ \\
& $\begin{array}{l}\text { 400X: } 32 \text { pictures named 613.400.01 - 32 (vertical scan bottom to } \\
\text { top) }\end{array}$ \\
& $\begin{array}{l}\text { 400X: } 2 \text { pictures named 613.400.roi1 (region of interesting } \\
\text { damage) and 613.400.scale (attempt to record zoom) }\end{array}$ \\
\hline
\end{tabular}

\section{Experiment 615:}

\begin{tabular}{|c|c|c|c|}
\hline Shot number: & 615 & Capsule dimensions: & $40 \mathrm{~mm} \times 27.0 \mathrm{~mm}$ \\
\hline Sample diameter: & $22.4 \mathrm{~mm}$ & Capsule front face: & $3 \mathrm{~mm}$ \\
\hline Sample thickness: & $5.0 \mathrm{~mm}$ & Capsule material: & Aluminum 6061 \\
\hline Sample condition: & Water-saturated & Ring material & Aluminum 6061 \\
\hline Flyer plate material: & Aluminum 1100 & Gas breech pressure: & $13.01 \mathrm{MPa}$ \\
\hline Flyer plate diameter: & $32 \mathrm{~mm}$ & Barrel diameter: & $35 \mathrm{~mm}$ \\
\hline Flyer plate thickness: & $3.00 \mathrm{~mm}$ & Gap behind flyer: & yes \\
\hline Flyer plate weight: & $6.56 \mathrm{~g}$ & X-ray taken: & yes \\
\hline Projectile material: & Lexan & Laser taken: & yes \\
\hline Projectile weight: & $33.7 \mathrm{~g}$ & Impact pressure: & $6.4 \mathrm{GPa}$ \\
\hline Projectile velocity & $800 \mathrm{~m} / \mathrm{s}$ & Date: & 11/06/1998 \\
\hline Comments: & \multicolumn{3}{|c|}{ 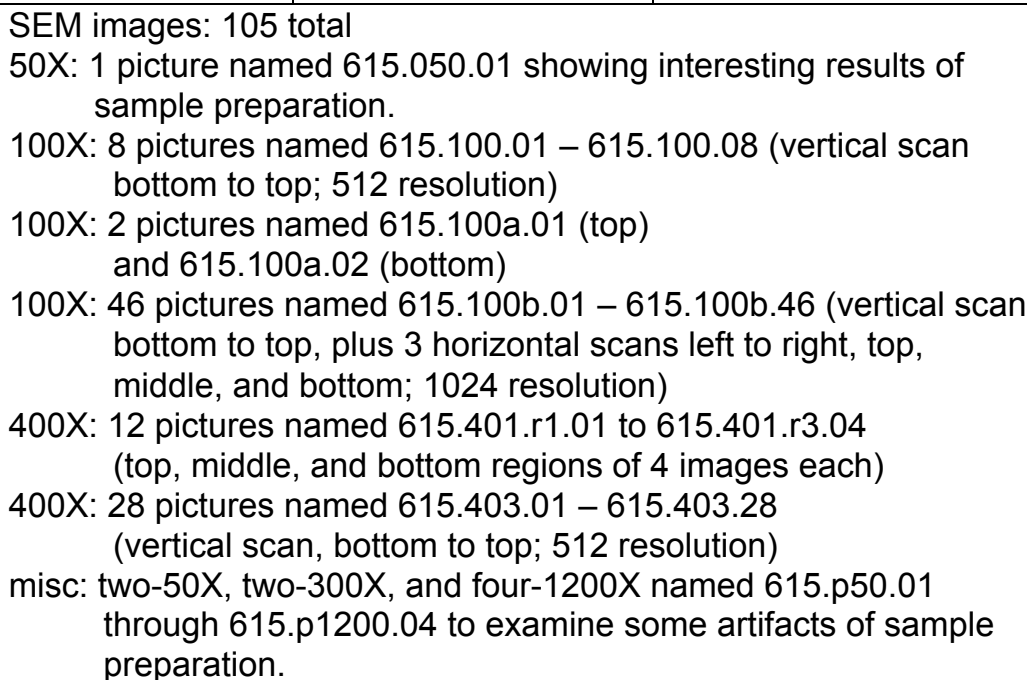 } \\
\hline
\end{tabular}




\section{Experiment 616:}

\begin{tabular}{|l|l|l|l|}
\hline Shot number: & 616 & Capsule dimensions: & $40 \mathrm{~mm}$ X 27.0 mm \\
\hline Sample diameter: & $22.4 \mathrm{~mm}$ & Capsule front face: & $5 \mathrm{~mm}$ \\
\hline Sample thickness: & $5.0 \mathrm{~mm}$ & Capsule material: & Aluminum 6061 \\
\hline Sample condition: & Water-pressurized & Ring material & Aluminum 6061 \\
\hline Flyer plate material: & Tantalum & Gas breech pressure: & $17.23 \mathrm{MPa}$ \\
\hline Flyer plate diameter: & $32 \mathrm{~mm}$ & Barrel diameter: & $35 \mathrm{~mm}$ \\
\hline Flyer plate thickness: & $3.0 \mathrm{~mm}$ & Gap behind flyer: & no \\
\hline Flyer plate weight: & $47.9 \mathrm{~g}$ & X-ray taken: & yes \\
\hline Projectile material: & Lexan & Laser taken: & yes \\
\hline Projectile weight: & $69.0 \mathrm{~g}$ & Impact pressure: & $8.6 \mathrm{GPa}$ \\
\hline Projectile velocity & $830 \mathrm{~m} / \mathrm{s}$ & Date: & $11 / 06 / 1998$ \\
\hline Comments: & $\begin{array}{l}\text { The sample was water-pressurized to 7.14 GPa using the hydraulic } \\
\text { cylinder. } \\
\text { SEM pictures: } 6 \text { total } \\
\text { 100X: 4 pictures named 616.100.01- 04 (top to bottom) } \\
\text { 400X: 2 pictures impact side named 616.400.top1-2 }\end{array}$ \\
\hline
\end{tabular}

\section{Experiment 677:}

\begin{tabular}{|c|c|c|c|}
\hline Shot number: & 677 & Capsule diameter: & $40 \mathrm{~mm} \times 37.8 \mathrm{~mm}$ \\
\hline Sample diameter: & $25.2 \mathrm{~mm}$ & Capsule front face: & $7 \mathrm{~mm}$ \\
\hline Sample thickness: & $23.0 \mathrm{~mm}$ & Capsule material: & Aluminum 6061 \\
\hline Sample condition: & Dry & Ring material & N/A \\
\hline Flyer plate material: & Aluminum 1100 & Gas breech pressure: & $18.61 \mathrm{MPa}$ \\
\hline Flyer plate diameter: & $32 \mathrm{~mm}$ & Barrel diameter: & $35 \mathrm{~mm}$ \\
\hline Flyer plate thickness: & $6.18 \mathrm{~mm}$ & Gap behind flyer: & yes \\
\hline Flyer plate weight: & $13.56 \mathrm{~g}$ & X-ray taken: & yes \\
\hline Projectile material: & Lexan & Laser taken: & yes \\
\hline Projectile weight: & $38.27 \mathrm{~g}$ & Impact pressure: & $7.1 \mathrm{GPa}$ \\
\hline Projectile velocity & $870 \mathrm{~m} / \mathrm{s}$ & Date: & $04 / 30 / 1999$ \\
\hline Comments: & \multicolumn{3}{|c|}{$\begin{array}{l}\text { Dry quartz sand experiment with a grain size }>212 \mu \mathrm{m}<250 \mu \mathrm{m} \text {, } \\
\text { porosity } 36 \%, 8.017 \mathrm{~cm}^{3} \text { sand in capsule. } \\
\text { SEM pictures: } 54 \text { total } \\
\text { 100X: } 9 \text { pictures named } 677.100 .01-09 \text { (vertical scan top to } \\
\text { bottom) } \\
\text { 400X: } 5 \text { pictures named } 677.402 .01-05 \\
\quad 27 \text { pictures named } 677.401 .06-33 \\
\quad 4 \text { pictures impact side named } 677.400 . \text { top } 1-4 \\
\quad 4 \text { pictures middle part named } 677.400 \text {.mid1-4 } \\
\quad 4 \text { pictures back side named } 677.400 . \text { bot } 1-4\end{array}$} \\
\hline
\end{tabular}




\section{Experiment 692:}

\begin{tabular}{|c|c|c|c|}
\hline Shot number: & 692 & Capsule diameter: & $40 \mathrm{~mm} \times 33.8 \mathrm{~mm}$ \\
\hline Sample diameter: & $25.2 \mathrm{~mm}$ & Capsule front face: & $7 \mathrm{~mm}$ \\
\hline Sample thickness: & $14.9 \mathrm{~mm}$ & Capsule material: & Aluminum 6061 \\
\hline Sample condition: & Water-pressurized & Ring material & Aluminum 6061 \\
\hline Flyer plate material: & Aluminum 1100 & Gas breech pressure: & 3.6 MPa \\
\hline Flyer plate diameter: & $32 \mathrm{~mm}$ & Barrel diameter: & $35 \mathrm{~mm}$ \\
\hline Flyer plate thickness: & $6.26 \mathrm{~mm}$ & Gap behind flyer: & yes \\
\hline Flyer plate weight: & $13.63 \mathrm{~g}$ & X-ray taken: & yes \\
\hline Projectile material: & Lexan & Laser taken: & yes \\
\hline Projectile weight: & $40.91 \mathrm{~g}$ & Impact pressure: & $2.7 \mathrm{GPa}$ \\
\hline Projectile velocity & $360 \mathrm{~m} / \mathrm{s}$ & Date: & 09/23/1999 \\
\hline Comments: & \multicolumn{3}{|c|}{$\begin{array}{l}\text { The sample was water-pressurized to } 7.79 \text { GPa using the hydraulic } \\
\text { cylinder. } \\
\text { SEM pictures: } 73 \text { total } \\
\text { 100X: } 10 \text { pictures named } 692.100 .01-10 \text { (vertical scan top to bot.) } \\
\text { 100X: } 3 \text { pictures named } 692.100 \text { bot }, 100 \text { mid, and } 100 \text { top } \\
\text { 400X: } 42 \text { pictures named } 692.400 .01-42 \\
4 \text { pictures impact side named } 692.400 . \text { top1- } 4 \\
4 \text { pictures middle part named } 692.400 \text {.mid1-4 } \\
4 \text { pictures back side named } 692.400 . \text { bot1-4 } \\
2 \text { pictures impact side named } 692.400 . \text { top1-2 } \\
3 \text { pictures middle part named } 692.402 \text {.mid1-3 } \\
2 \text { pictures back side named } 692.402 \text { bot } 1-2\end{array}$} \\
\hline
\end{tabular}

\section{Experiment 693:}

\begin{tabular}{|c|c|c|c|}
\hline Shot number: & 693 & Capsule diameter: & $40 \mathrm{~mm} \times 33.8 \mathrm{~mm}$ \\
\hline Sample diameter: & $25.2 \mathrm{~mm}$ & Capsule front face: & $7 \mathrm{~mm}$ \\
\hline Sample thickness: & $14.8 \mathrm{~mm}$ & Capsule material: & Aluminum 6061 \\
\hline Sample condition: & Water-saturated & Ring material & Aluminum 6061 \\
\hline Flyer plate material: & Aluminum 1100 & Gas breech pressure: & $4.07 \mathrm{MPa}$ \\
\hline Flyer plate diameter: & $32 \mathrm{~mm}$ & Barrel diameter: & $35 \mathrm{~mm}$ \\
\hline Flyer plate thickness: & $6.25 \mathrm{~mm}$ & Gap behind flyer: & yes \\
\hline Flyer plate weight: & $13.61 \mathrm{~g}$ & X-ray taken: & yes \\
\hline Projectile material: & Lexan & Laser taken: & yes \\
\hline Projectile weight: & $40.56 \mathrm{~g}$ & Impact pressure: & $3.8 \mathrm{GPa}$ \\
\hline Projectile velocity & $490 \mathrm{~m} / \mathrm{s}$ & Date: & 09/23/1999 \\
\hline Comments: & \multicolumn{3}{|c|}{$\begin{array}{l}\text { SEM pictures: } 62 \text { total } \\
\text { 100X: } 10 \text { pictures named } 693.100 .01-10 \\
\text { 400X: } 40 \text { pictures named } 693.400 .01-40 \\
4 \text { pictures impact side named } 693.400 . \text { top } 1-4 \\
4 \text { pictures middle part named } 693.400 . \text { mid1-4 } \\
4 \text { pictures back side named } 693.400 . \text { bot } 1-4\end{array}$} \\
\hline
\end{tabular}




\section{Experiment 696:}

\begin{tabular}{|c|c|c|c|}
\hline Shot number: & 696 & Capsule diameter: & $40 \mathrm{~mm} \times 33.8 \mathrm{~mm}$ \\
\hline Sample diameter: & $25.2 \mathrm{~mm}$ & Capsule front face: & $3 \mathrm{~mm}$ \\
\hline Sample thickness: & $14.9 \mathrm{~mm}$ & Capsule material: & Aluminum 6061 \\
\hline Sample condition: & Dry & Ring material & Aluminum 6061 \\
\hline Flyer plate material: & Aluminum 1100 & Gas breech pressure: & $3.65 \mathrm{MPa}$ \\
\hline Flyer plate diameter: & $32 \mathrm{~mm}$ & Barrel diameter: & $35 \mathrm{~mm}$ \\
\hline Flyer plate thickness: & $6.20 \mathrm{~mm}$ & Gap behind flyer: & yes \\
\hline Flyer plate weight: & $13.46 \mathrm{~g}$ & X-ray taken: & yes \\
\hline Projectile material: & Lexan & Laser taken: & yes \\
\hline Projectile weight: & $40.40 \mathrm{~g}$ & Impact pressure: & $3.5 \mathrm{GPa}$ \\
\hline Projectile velocity & $450 \mathrm{~m} / \mathrm{s}$ & Date: & 05/26/1999 \\
\hline Comments: & \multicolumn{3}{|c|}{$\begin{array}{l}\text { SEM pictures: } 60 \text { total } \\
\text { 100X: } 10 \text { pictures named } 696.100 .01-10 \\
\text { 400X: } 38 \text { pictures named } 696.401 .01-38 \\
4 \text { pictures impact side named } 696.400 . \text { top } 1-4 \\
4 \text { pictures middle part named } 696.400 . \text { mid1-4 } \\
4 \text { pictures back side named } 696.400 . \text { bot1-4 }\end{array}$} \\
\hline
\end{tabular}

\section{Experiment 697:}

\begin{tabular}{|c|c|c|c|}
\hline Shot number: & 697 & Capsule diameter: & $40 \mathrm{~mm} \times 33.8 \mathrm{~mm}$ \\
\hline Sample diameter: & $25.2 \mathrm{~mm}$ & Capsule front face: & $3 \mathrm{~mm}$ \\
\hline Sample thickness: & $15.0 \mathrm{~mm}$ & Capsule material: & Aluminum 6061 \\
\hline Sample condition: & Dry & Ring material & Aluminum 6061 \\
\hline Flyer plate material: & PMMA & Gas breech pressure: & $4.17 \mathrm{MPa}$ \\
\hline Flyer plate diameter: & $32 \mathrm{~mm}$ & Barrel diameter: & $35 \mathrm{~mm}$ \\
\hline Flyer plate thickness: & $6.12 \mathrm{~mm}$ & Gap behind flyer: & yes \\
\hline Flyer plate weight: & $5.84 \mathrm{~g}$ & X-ray taken: & yes \\
\hline Projectile material: & Lexan & Laser taken: & yes \\
\hline Projectile weight: & $33.01 \mathrm{~g}$ & Impact pressure: & $1.4 \mathrm{GPa}$ \\
\hline Projectile velocity & $450 \mathrm{~m} / \mathrm{s}$ & Date: & $06 / 08 / 1999$ \\
\hline Comments: & \multicolumn{3}{|c|}{$\begin{array}{l}\text { SEM pictures: } 69 \text { total } \\
\text { 100X: } 10 \text { pictures named } 697.100 .01-10 \\
3 \text { pictures named } 697.100 \text { bot, } 697.100 \mathrm{mid}, 697.100 \text { top } \\
\text { 400X: } 39 \text { pictures named } 697.401 .01-39 \\
4 \text { pictures impact side named } 697.400 . \text { top } 1-4 \\
4 \text { pictures middle part named } 697.400 . \text { mid1-4 } \\
4 \text { pictures back side named } 697.400 . \text { bot1-4 } \\
5 \text { named } 697.402 \text { bot.01-02, } 697.402 \text { mid.01, } \\
697.402 \text { top.01-02 }\end{array}$} \\
\hline
\end{tabular}




\section{Experiment 698:}

\begin{tabular}{|l|l|l|l|}
\hline Shot number: & 698 & Capsule diameter: & $40 \mathrm{~mm}$ X 33.8 mm \\
\hline Sample diameter: & $25.2 \mathrm{~mm}$ & Capsule front face: & $3 \mathrm{~mm}$ \\
\hline Sample thickness: & $14.97 \mathrm{~mm}$ & Capsule material: & Aluminum 6061 \\
\hline Sample condition: & Dry & Ring material & Aluminum 6061 \\
\hline Flyer plate material: & OFHC Copper & Gas breech pressure: & $19.03 \mathrm{MPa}$ \\
\hline Flyer plate diameter: & $32 \mathrm{~mm}$ & Barrel diameter: & $35 \mathrm{~mm}$ \\
\hline Flyer plate thickness: & $6.21 \mathrm{~mm}$ & Gap behind flyer: & yes \\
\hline Flyer plate weight: & $44.35 \mathrm{~g}$ & X-ray taken: & yes \\
\hline Projectile material: & Lexan & Laser taken: & yes \\
\hline Projectile weight: & $71.26 \mathrm{~g}$ & Impact pressure: & $7.8 \mathrm{GPa}$ \\
\hline Projectile velocity & $680 \mathrm{~m} / \mathrm{s}$ & Date: & 06/08/1999 \\
\hline Comments: & $\begin{array}{l}\text { Front-part of the capsule came off. The flyer plate sealed the } \\
\text { opening and therefore the sandstone target could be recovered. } \\
\text { SEM pictures: 10 total } \\
\text { 100X: 2 pictures named 698.100.bot, 698.100.top } \\
\text { 400X: 8 pictures named 698.401.bot1-4, 698.401.top1-4 }\end{array}$ \\
\hline
\end{tabular}

\section{Experiment 700:}

\begin{tabular}{|c|c|c|c|}
\hline Shot number: & 700 & Capsule diameter: & $40 \mathrm{~mm} \times 33.8 \mathrm{~mm}$ \\
\hline Sample diameter: & $25.2 \mathrm{~mm}$ & Capsule front face: & $7 \mathrm{~mm}$ \\
\hline Sample thickness: & $14.8 \mathrm{~mm}$ & Capsule material: & Aluminum 6061 \\
\hline Sample condition: & Water-pressurized & Ring material & Aluminum 6061 \\
\hline Flyer plate material: & Aluminum 1100 & Gas breech pressure: & $15.86 \mathrm{MPa}$ \\
\hline Flyer plate diameter: & $32 \mathrm{~mm}$ & Barrel diameter: & $35 \mathrm{~mm}$ \\
\hline Flyer plate thickness: & $6.30 \mathrm{~mm}$ & Gap behind flyer: & yes \\
\hline Flyer plate weight: & $13.66 \mathrm{~g}$ & X-ray taken: & yes \\
\hline Projectile material: & Lexan & Laser taken: & yes \\
\hline Projectile weight: & $40.78 \mathrm{~g}$ & Impact pressure: & $6.4 \mathrm{GPa}$ \\
\hline Projectile velocity & $793 \mathrm{~m} / \mathrm{s}$ & Date: & 06/08/1999 \\
\hline Comments: & \multicolumn{3}{|c|}{ 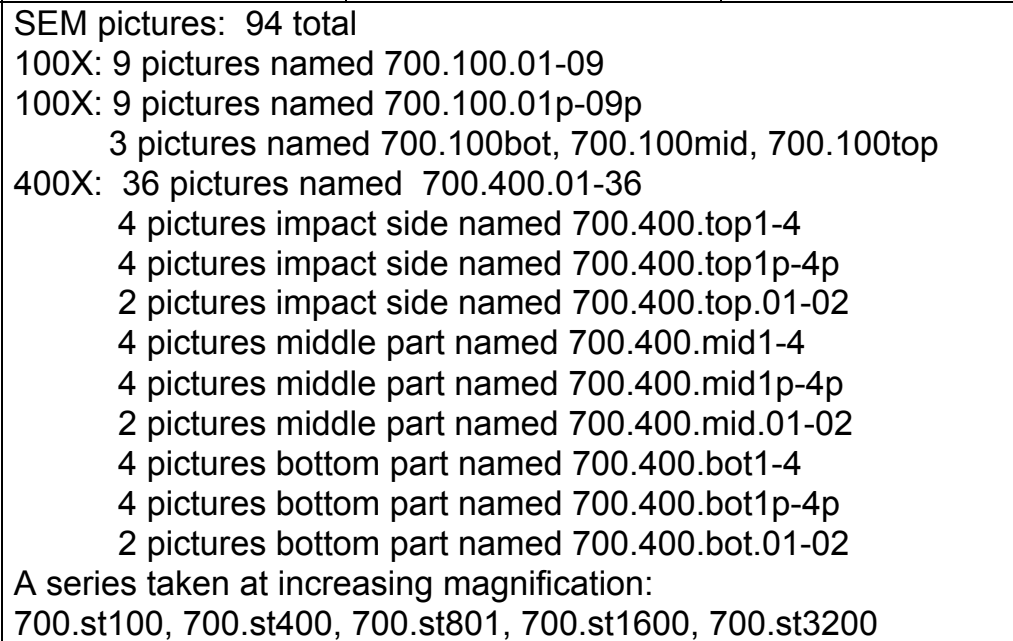 } \\
\hline
\end{tabular}




\section{Experiment 701:}

\begin{tabular}{|c|c|c|c|}
\hline Shot number: & 701 & Capsule diameter: & $40 \mathrm{~mm} \times 33.8 \mathrm{~mm}$ \\
\hline Sample diameter: & $25.2 \mathrm{~mm}$ & Capsule front face: & $3 \mathrm{~mm}$ \\
\hline Sample thickness: & $14.9 \mathrm{~mm}$ & Capsule material: & Aluminum 6061 \\
\hline Sample condition: & Water-pressurized & Ring material & Aluminum 6061 \\
\hline Flyer plate material: & PMMA & Gas breech pressure: & $4.13 \mathrm{MPa}$ \\
\hline Flyer plate diameter: & $32 \mathrm{~mm}$ & Barrel diameter: & $35 \mathrm{~mm}$ \\
\hline Flyer plate thickness: & $6.14 \mathrm{~mm}$ & Gap behind flyer: & yes \\
\hline Flyer plate weight: & $5.76 \mathrm{~g}$ & X-ray taken: & yes \\
\hline Projectile material: & Lexan & Laser taken: & yes \\
\hline Projectile weight: & $32.78 \mathrm{~g}$ & Impact pressure: & $1.3 \mathrm{GPa}$ \\
\hline Projectile velocity & $438 \mathrm{~m} / \mathrm{s}$ & Date: & 06/10/1999 \\
\hline Comments: & \multicolumn{3}{|c|}{$\begin{array}{l}\text { The sample was water-pressurized to } 7.65 \mathrm{GPa} \text { using the } \\
\text { hydraulic cylinder. } \\
\text { SEM pictures: } 64 \text { total } \\
\text { 100X: } 11 \text { pictures named } 701.100 .01-11 \\
\text { 400X: } 41 \text { pictures named } 701.400 .01-41 \\
\quad 4 \text { pictures impact side named } 701.400 . \text { top } 1-4 \\
\quad 4 \text { pictures middle part named } 701.400 \text {.mid1-4 } \\
\quad 4 \text { pictures back side named } 701.400 . \text { bot } 1-4\end{array}$} \\
\hline
\end{tabular}

\section{Experiment 739:}

\begin{tabular}{|c|c|c|c|}
\hline Shot number: & 739 & Capsule diameter: & $40 \mathrm{~mm} \times 33.8 \mathrm{~mm}$ \\
\hline Sample diameter: & $25.2 \mathrm{~mm}$ & Capsule front face: & $7 \mathrm{~mm}$ \\
\hline Sample thickness: & $14.9 \mathrm{~mm}$ & Capsule material: & Aluminum 6061 \\
\hline Sample condition: & Water-saturated & Ring material & Aluminum 6061 \\
\hline Flyer plate material: & PMMA & Gas breech pressure: & $4.13 \mathrm{MPa}$ \\
\hline Flyer plate diameter: & $32 \mathrm{~mm}$ & Barrel diameter: & $35 \mathrm{~mm}$ \\
\hline Flyer plate thickness: & $5.93 \mathrm{~mm}$ & Gap behind flyer: & yes \\
\hline Flyer plate weight: & $5.62 \mathrm{~g}$ & X-ray taken: & yes \\
\hline Projectile material: & Lexan & Laser taken: & yes \\
\hline Projectile weight: & $35.01 \mathrm{~g}$ & Impact pressure: & 1.2 GPa \\
\hline Projectile velocity & $412 \mathrm{~m} / \mathrm{s}$ & Date: & $08 / 26 / 1999$ \\
\hline Comments: & \multicolumn{3}{|c|}{$\begin{array}{l}\text { SEM pictures: } 62 \text { total } \\
\text { 100X: } 10 \text { pictures named } 739.100 .01-10 \\
\text { 400X: } 41 \text { pictures named } 739.400 .01-40 \\
4 \text { pictures impact side named } 739.400 . \text { top1-4 } \\
4 \text { pictures middle part named } 739.400 . \text { mid1-4 } \\
4 \text { pictures back side named } 739.400 . \text { bot } 1-4\end{array}$} \\
\hline
\end{tabular}




\section{Experiment 740:}

\begin{tabular}{|c|c|c|c|}
\hline Shot number: & 740 & Capsule diameter: & $40 \mathrm{~mm} \times 27.2 \mathrm{~mm}$ \\
\hline Sample diameter: & $25.2 \mathrm{~mm}$ & Capsule front face: & $5 \mathrm{~mm}$ \\
\hline Sample thickness: & $7.97 \mathrm{~mm}$ & Capsule material: & Stainless steel \\
\hline Sample condition: & Water-saturated & Ring material & N/A \\
\hline Flyer plate material: & Aluminum 1100 & Gas breech pressure: & $15.81 \mathrm{MPa}$ \\
\hline Flyer plate diameter: & $32 \mathrm{~mm}$ & Barrel diameter: & $35 \mathrm{~mm}$ \\
\hline Flyer plate thickness: & $3.00 \mathrm{~mm}$ & Gap behind flyer: & yes \\
\hline Flyer plate weight: & $6.60 \mathrm{~g}$ & X-ray taken: & yes \\
\hline Projectile material: & Lexan & Laser taken: & yes \\
\hline Projectile weight: & $36.00 \mathrm{~g}$ & Impact pressure: & $7.6 \mathrm{GPa}$ \\
\hline Projectile velocity & $662 \mathrm{~m} / \mathrm{s}$ & Date: & 09/09/1999 \\
\hline Comments: & \multicolumn{3}{|c|}{$\begin{array}{l}\text { Quartz sand in water-saturated conditions } \\
\text { SEM pictures: } 52 \text { total } \\
\text { 100X: } 6 \text { pictures named } 740.100 .01-06 \\
\text { 400X: } 46 \text { pictures named } 740.401 .01-46 \\
\quad 2 \text { rows scanned, } 23 \text { images each row }\end{array}$} \\
\hline
\end{tabular}

\section{Experiment 741:}

\begin{tabular}{|c|c|c|c|}
\hline Shot number: & 741 & Capsule diameter: & $40 \mathrm{~mm} \times 27.2 \mathrm{~mm}$ \\
\hline Sample diameter: & $25.2 \mathrm{~mm}$ & Capsule front face: & $5 \mathrm{~mm}$ \\
\hline Sample thickness: & $7.97 \mathrm{~mm}$ & Capsule material: & Stainless steel \\
\hline Sample condition: & Dry & Ring material & N/A \\
\hline Flyer plate material: & Aluminum 1100 & Gas breech pressure: & $15.81 \mathrm{MPa}$ \\
\hline Flyer plate diameter: & $32 \mathrm{~mm}$ & Barrel diameter: & $35 \mathrm{~mm}$ \\
\hline Flyer plate thickness: & $3.02 \mathrm{~mm}$ & Gap behind flyer: & yes \\
\hline Flyer plate weight: & $6.65 \mathrm{~g}$ & X-ray taken: & yes \\
\hline Projectile material: & Lexan & Laser taken: & yes \\
\hline Projectile weight: & $35.91 \mathrm{~g}$ & Impact pressure: & $7.3 \mathrm{GPa}$ \\
\hline Projectile velocity & $640 \mathrm{~m} / \mathrm{s}$ & Date: & 09/10/1999 \\
\hline Comments: & \multicolumn{3}{|c|}{$\begin{array}{l}\text { Quartz sand in dry condition } \\
\text { SEM pictures: } 41 \text { total } \\
\text { 100X: } 5 \text { pictures named } 741.100 .01-05 \\
\text { 400X: } 36 \text { pictures named } 741.402 .01-36 \\
\quad 2 \text { rows scanned, } 18 \text { images each row }\end{array}$} \\
\hline
\end{tabular}




\section{Experiment 742:}

\begin{tabular}{|c|c|c|c|}
\hline Shot number: & 742 & Capsule diameter: & $40 \mathrm{~mm} \times 33.8 \mathrm{~mm}$ \\
\hline Sample diameter: & $25.2 \mathrm{~mm}$ & Capsule front face: & $7 \mathrm{~mm}$ \\
\hline Sample thickness: & $14.9 \mathrm{~mm}$ & Capsule material: & Aluminum 6061 \\
\hline Sample condition: & Gas-pressurized & Ring material & Aluminum 6061 \\
\hline Flyer plate material: & Aluminum 1100 & Gas breech pressure: & $15.81 \mathrm{MPa}$ \\
\hline Flyer plate diameter: & $32 \mathrm{~mm}$ & Barrel diameter: & $35 \mathrm{~mm}$ \\
\hline Flyer plate thickness: & $6.23 \mathrm{~mm}$ & Gap behind flyer: & yes \\
\hline Flyer plate weight: & $13.56 \mathrm{~g}$ & X-ray taken: & yes \\
\hline Projectile material: & Lexan & Laser taken: & yes \\
\hline Projectile weight: & $42.91 \mathrm{~g}$ & Impact pressure: & $6.2 \mathrm{GPa}$ \\
\hline Projectile velocity & $770 \mathrm{~m} / \mathrm{s}$ & Date: & 09/13/1999 \\
\hline Comments: & \multicolumn{3}{|c|}{$\begin{array}{l}\text { The sample was gas-pressurized with helium to } 27.56 \mathrm{GPa} \text { using a } \\
\text { He-gas bottle. } \\
\text { SEM pictures: } 59 \text { total } \\
\text { 100X: } 10 \text { pictures named } 742.100 .01-10 \\
\text { 400X: } 37 \text { pictures named } 742.401 .01-37 \\
\quad 4 \text { pictures impact side named } 742.401 \text {.top1-4 } \\
\quad 4 \text { pictures middle part named } 742.401 \text {.mid1-4 } \\
4 \text { pictures back side named } 742.401 \text {.bot1-4 }\end{array}$} \\
\hline
\end{tabular}

\section{Experiment 743:}

\begin{tabular}{|c|c|c|c|}
\hline Shot number: & 742 & Capsule diameter: & $40 \mathrm{~mm} \times 33.8 \mathrm{~mm}$ \\
\hline Sample diameter: & $25.2 \mathrm{~mm}$ & Capsule front face: & $7 \mathrm{~mm}$ \\
\hline Sample thickness: & $15.1 \mathrm{~mm}$ & Capsule material: & Aluminum 6061 \\
\hline Sample condition: & Gas-pressurized & Ring material & Aluminum 6061 \\
\hline Flyer plate material: & Aluminum 1100 & Gas breech pressure: & $3.65 \mathrm{MPa}$ \\
\hline Flyer plate diameter: & $32 \mathrm{~mm}$ & Barrel diameter: & $35 \mathrm{~mm}$ \\
\hline Flyer plate thickness: & $6.25 \mathrm{~mm}$ & Gap behind flyer: & yes \\
\hline Flyer plate weight: & $13.60 \mathrm{~g}$ & X-ray taken: & yes \\
\hline Projectile material: & Lexan & Laser taken: & yes \\
\hline Projectile weight: & $43.16 \mathrm{~g}$ & Impact pressure: & $2.6 \mathrm{GPa}$ \\
\hline Projectile velocity & $348 \mathrm{~m} / \mathrm{s}$ & Date: & 09/14/1999 \\
\hline Comments: & \multicolumn{3}{|c|}{$\begin{array}{l}\text { The sample was gas-pressurized with helium to } 28.96 \text { GPa using a } \\
\text { He-gas bottle. } \\
\text { SEM pictures: } 64 \text { total } \\
\text { 100X: } 11 \text { pictures named } 743.100 .01-11 \\
\text { 400X: } 41 \text { pictures named } 743.402 .01-41 \\
\quad 4 \text { pictures impact side named } 743.402 \text {.top } 1-4 \\
\quad 4 \text { pictures middle part named } 743.402 \text {. mid1-4 } \\
4 \text { pictures back side named } 743.402 \text {.bot } 1-4\end{array}$} \\
\hline
\end{tabular}




\section{Experiment 744: Berea Sandstone}

\begin{tabular}{|c|c|c|c|}
\hline Shot number: & 744 & Capsule diameter: & $40 \mathrm{~mm} \times 33.8 \mathrm{~mm}$ \\
\hline Sample diameter: & $25.2 \mathrm{~mm}$ & Capsule front face: & $7 \mathrm{~mm}$ \\
\hline Sample thickness: & $14.9 \mathrm{~mm}$ & Capsule material: & Aluminum 6061 \\
\hline Sample condition: & Gas-pressurized & Ring material & Aluminum 6061 \\
\hline Flyer plate material: & PMMA & Gas breech pressure: & 4.14 MPa \\
\hline Flyer plate diameter: & $32 \mathrm{~mm}$ & Barrel diameter: & $35 \mathrm{~mm}$ \\
\hline Flyer plate thickness: & $6.02 \mathrm{~mm}$ & Gap behind flyer: & yes \\
\hline Flyer plate weight: & $5.69 \mathrm{~g}$ & X-ray taken: & yes \\
\hline Projectile material: & Lexan & Laser taken: & yes \\
\hline Projectile weight: & $34.82 \mathrm{~g}$ & Impact pressure: & $1.3 \mathrm{GPa}$ \\
\hline Projectile velocity & $430 \mathrm{~m} / \mathrm{s}$ & Date: & 09/14/1999 \\
\hline Comments: & \multicolumn{3}{|c|}{$\begin{array}{l}\text { The sample was gas-pressurized with helium to } 27.56 \mathrm{GPa} \text { using a } \\
\text { He-gas bottle. } \\
\text { SEM pictures: } 63 \text { total } \\
\text { 100X: } 10 \text { pictures named } 744.100 .01-10 \\
\text { 400X: } 41 \text { pictures named } 744.401 .01-41 \\
\quad 4 \text { pictures impact side named } 744.401 . \text { top } 1-4 \\
\quad 4 \text { pictures middle part named } 744.401 \text {.mid1-4 } \\
\quad 4 \text { pictures back side named } 744.401 \text {.bot1-4 }\end{array}$} \\
\hline
\end{tabular}

\section{Acknowledgements}

We gratefully acknowledge Samuel B. Weaver and William J. Metz Jr. for performing the gas-gun experiments. Erikk A. Ojala, Steven J. Caldwell, James G. Van Lewen and Ronald J. Pletcher provided invaluable technical support. We also thank Marjorie G. Snow for guidance at the SEM and Phillip M. Halleck at Pennsylvania State University for performing the laser particle size analysis. This work was performed at Lawrence Livermore National Laboratory under the auspices of the U.S. Department of Energy under contract No. W-7405-Eng-48 and at Los Alamos National Laboratory under contract No. W-7405-Eng-36.

\section{References}

Hagelberg, C. R., Swift, R. P., Carney, T. C., Greening, D., Hiltl, M. and Nellis, W. J. (2000a): Modeling Shock Recovery Experiments of Sandstone, in Shock Compression in Condensed Matter-1999, edited by M. D. Furnish, AIP Conference Proceedings.

Hagelberg, C. R., Hiltl, M., Swift, R. P. and Nellis, W. J. (2000b): Analysis of Shock Damage to Berea Sandstone Using SEM Images of Samples from Impact Recovery Experiments, Proceedings ASME/ETCE 2000 Joint Conference on Energy for the New Millennium, Feb. 14-17, 2000, New Orleans, LA, USA.

Hagelberg, C. R., M. Hiltl, R. P. Swift, K. W. Jones, and H. Feng (2000c): X-Ray Computed Micro Tomography of Shock-Recovered Berea Sandstone, Los Alamos Report in progress.

Halleck, P. M. (1996): Lab Tests Expand Knowledge of Fractured Reservoir Perforating Damage, GasTips, 2, 39-42.

Halleck, P.M. (1997): Recent Advances in Understanding Perforator Penetration and Flow Performance, SPE Drilling \& Completion, 12, 19-26. 
Hiltl, M., Swift, R. P., Hagelberg, C. R., Carney, T. C. and Nellis, W. J. (2000a): Shock-recovery Experiments of Sandstone under Dry and Water-saturated Conditions, in Shock Compression in Condensed Matter-1999, edited by M. D. Furnish, AIP Conference Proceedings.

Hiltl, M., Hagelberg, C. R., Swift, R. P., Carney T. C. and Nellis, W. J. (2000b): Dynamic Response of Berea Sandstone Shock Loaded Under Dry, Wet, and Water-pressurized Conditions. Proceedings, International Association for the Advancement of High Pressure Science and Technology (AIRAPT) International Conference on High Pressure Science and Technology (AIRAPT-17), July 25 -July 30, 1999, University of Hawaii, Honolulu, Hawaii, USA.

Hiltl, M., Hagelberg, C. R., Swift, R. P. and Nellis, W. J. (2000c): Shock-Recovery Experiments of Berea Sandstone, submitted to Journal of Rock Mechanics and Rock Engineering.

Krueger, R. F. (Feb. 1986): An Overview of Fragmentation Damage and Well Productivity in Oilfield Operations, J. Pet. Tech. 131-152.

Lichtenberger, A. (1995): Ductile behavior of some materials in the shaped charge jet, in: Metallurgical and Materials Applications of Shock-Wave and High-Strain-Rate Phenomena (EXPLOMET95), ed. Murr, L. E; Staudhammer, K. P. and Meyers, M. A., Elsevier Science B. V., Chapter 55, pp. 463-470.

Lide, D. R. (1997): Handbook of Chemistry and Physics, $78^{\text {th }}$ Edition, CRC Press, Boca Roton, New York

Lundergan, C. D., Herrmann, W. (1963): Equation of State of 6061-T6 Aluminum at Low Pressures, J. Appl. Phys. 34, 2046-2052

Marsh, S. P. (1979): Los Alamos Hugoniot Data, University of Berkeley Press.

McLeod, H. O. (1983): The Effect of Perforating Conditions on Well Performance, J. Pet. Tech., 31-36.

Mitchell, A. C., Nellis, W. J. (1981): Shock Compression of Aluminum, Copper, and Tantalum, J. Appl. Phys. 52, 3363-3374.

Rice, M. H., McQueen, R. G., Walsh, J. M. (1958): Compression of Solids by Strong Shock Waves, Solid State Physics, 6, 1-63.

Swift, R. P., Behrmann, L. A., Halleck, P. M. and Krogh, K. E. (1998): Micro-Mechanical Modeling of Perforating Shock Damage, SPE paper 39458, SPE Proc. of Int. Sym. on Formation Damage Control, 381.

Swift, R. P., Hagelberg, C. R., Carney, T. C., Greening, D. and Hiltl, M. (2000a): Modeling StressInduced Damage from Impact Recovery Experiments, Proceedings ASME/ETCE 2000 Joint Conference on Energy for the New Millennium, Feb. 14-17, 2000, New Orleans, LA, USA.

Swift, R. P., Hagelberg, C. R. and Hiltl, M. (2000b): A Numerical Analysis of Stress Wave-Induced Damage of Sandstone for Dry and Saturated Conditions. Fourth North American Rock Mechanics Symposium, July 31- Aug 3, 2000 - University of Washington - Seattle, WA. USA. 